\title{
GESTIÓN DE LA COMUNICACIÓN EN LAS UNIVERSIDADES PÚBLICAS ESPAÑOLAS
}

\author{
Communication management in Spanish \\ public universities
}

\section{Esther Simancas-González y Marcial García-López}

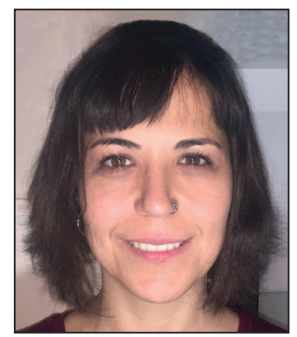

Esther Simancas-González es doctora en Comunicación Audiovisual y Publicidad y licenciada en Publicidad y Relaciones Públicas por la Universidad de Málaga. Es docente del Departamento de Marketing y Comunicación de la Universidad de Cádiz e investigadora del Instituto Universitario para el Desarrollo Social Sostenible (Indess). Pertenece al grupo de investigación HUM619: Profesorado, Comunicación e Investigación Educativa.

http://orcid.org/0000-0003-0747-8210

Universidad de Cádiz, Facultad de Ciencias Sociales y de la Comunicación Av. de la Universidad, s/n. 11405 Jerez de la Frontera (Cádiz), España esther.simancas@uca.es

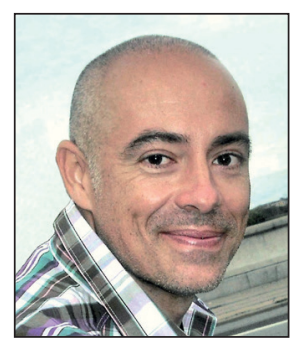

Marcial García-López es doctor en Ciencias de la Información y licenciado en Publicidad y Relaciones Públicas por la Universidad Complutense de Madrid. Profesor titular en la Facultad de Ciencias de la Comunicación de la Universidad de Málaga, pertenece al grupo de investigación HUM619: Profesorado, Comunicación e Investigación Educativa y es coordinador del proyecto Comsolidar: Comunicación Solidaria. http://orcid.org/0000-0002-1668-3526

Universidad de Málaga, Facultad de Ciencias de la Comunicación Calle León Tolstoi, s/n. 29010 Málaga, España mgarcialo@uma.es

\section{Resumen}

Este trabajo analiza la gestión de la comunicación institucional en las universidades públicas españolas, a partir de la realización de entrevistas a los dircoms universitarios. Los resultados muestran que la tendencia de la política comunicativa de las universidades se dirige hacia la creación de marcas universitarias diferenciadas, con el fin de alcanzar un posicionamiento que les permita ser atractivas y competitivas en el mercado. Sin embargo, además de las carencias detectadas en la gestión de la comunicación en las universidades públicas, lo que pone en riesgo el logro de ese objetivo, se evidencia el modelo difusionista como el dominante, opacando modelos alternativos, más democratizadores de la institución.

\section{Palabras clave}

Comunicación universitaria; Comunicación institucional; Comunicación participativa; Comunicación pública; Estrategias de comunicación; Gestión de la comunicación; Dircoms; Universidad pública; Universidad española.

\section{Abstract}

This paper analyzes communication management in Spanish public universities by interviewing communication directors of these institutions. Results show that, in addition to the shortcomings identified in the management of communications in public universities, which undermines the achievement of the exact marketing objective, the diffusional model is dominant, playing a negative influencing role. The diffusional model obscures other alternative models that would show more democratic institutions.

\section{Keywords}

University communication; Institutional Communication; Participatory communication; Public communication; Communication strategies; Communication management; Dircoms; Public universities; Spanish universities.

Simancas-González, Esther; García-López, Marcial (2017). “Gestión de la comunicación en las universidades públicas españolas". El profesional de la información, v. 26, n. 4, pp. 735-744. 


\section{La universidad y la comunicación}

Las transformaciones sociales, culturales, políticas y económicas, aceleradas con las innovaciones tecnológicas, confieren especial protagonismo a la comunicación organizacional (Túñez-López, 2012). Ello provoca que en el momento actual la supervivencia de las organizaciones sociales esté condicionada en gran parte por la imagen y la confianza que logren generar.

Las universidades públicas en España no son ajenas a esta coyuntura. Se puede afirmar que en todas existe un departamento que se encarga de gestionar la comunicación, situado por lo general en las más altas instancias universitarias, lo que denota el grado de institucionalización que ha alcanzado la comunicación. No obstante, el grueso de las investigaciones en este tema (Losada-Vázquez, 1998; Losada-Díaz, 2002; 2004), confirman que la universidad no ha sido capaz de dotarse de mecanismos eficaces para su gestión.

Las estrategias comunicativas de las universidades públicas españolas están centradas en la proyección de imagen, con el fin de ser atrayentes y competitivas en el mercado

Un problema endémico de la universidad al que hacen referencia diversos autores es la comunicación interna (Fernández-Beltrán, 2007; Palencia-Lefler-Ors, 2008; Herranz-dela-Casa; Tapia-Frade; Vicente-Lázaro, 2009). Los factores más determinantes podrían ser:

- multiplicidad de actores que conforman la institución;

- preeminencia de canales descendentes y unidireccionales;

- falta de voluntad para que esto cambie;

- predominio de estrategias comunicacionales únicamente centradas en lo externo.

Investigaciones recientes demuestran el escaso aprovechamiento y versatilidad de las universidades en el entorno digital (De-Aguilera-Moyano; Farias-Batlle; Baraybar-Fernández, 2010; Paniagua-Rojano; Gómez-Calderón; Fernández-Sande, 2012; Paniagua-Rojano; Gómez-Calderón, 2012 Gómez-Calderón; Paniagua-Rojano, 2014; González-Díaz; Iglesias-García; Codina, 2015; López-Pérez; Olvera-Lobo, 2016).

En los últimos 15 años se ha producido una clara inclinación de las investigaciones sobre comunicación institucional universitaria hacia la importancia de la gestión de los intangibles, entendiéndose que la creación de marcas universitarias diferenciadas debe ser uno de los objetivos comunicacionales prioritarios, y la principal solución para afrontar el problema comunicativo de la universidad (Castillo-Díaz, 2007; Zamora-Medina; Hernández-Gómez, 2014; Losada-Díaz, 2004).

Es necesario que la universidad pública disponga de una buena capacidad de comunicación interna y externa, ya que es en esta institución donde reside y avanza el conocimiento científico y social. Sin embargo, la naturaleza pública, la sin- gular idiosincrasia y la estructura especialmente compleja de esta institución condicionan la formación de un modelo de comunicación específico, que esté orientado al impulso y la conformación de una universidad pública democrática y de dimensiones plurales. Es más, su propia legitimidad y supervivencia en el siglo XXI como espacio de aprendizaje, reflexión y generación de saberes dependerán en gran medida de su capacidad para implementar una gestión más abierta, inclusiva y transparente (Chomsky, 2013), que responda a los nuevos valores y marcos de relación impulsados por la comunicación en red (Simancas-González, 2016). ¿En qué medida esto es así? Resulta imprescindible realizar un diagnóstico del estado de la gestión de la comunicación en las universidades públicas en España.

\section{Objetivos de la investigación}

El objetivo general es analizar la gestión de la comunicación institucional llevada a cabo por los departamentos de comunicación de las universidades públicas españolas para detectar modelos dominantes y posibles enfoques alternativos. En concreto se busca:

1. Describir los objetivos de la comunicación universitaria.

2. Evaluar si en las universidades públicas españolas se realiza una gestión integral y planificada de la comunicación.

3. Analizar el funcionamiento de los departamentos de comunicación y el rol de los responsables de comunicación de las universidades públicas.

4. Identificar los públicos prioritarios y los principales canales de la comunicación universitaria.

\section{Material y métodos}

El objeto de estudio está compuesto por 47 universidades públicas españolas ${ }^{1}$, al excluir las de carácter especial ${ }^{2}$ para prever posibles inferencias que pudiesen tergiversar los resultados del estudio.

\section{El modelo de comunicación dominante en las universidades públicas españolas es el difusionista y vertical, obviando modelos alternativos, más participativos y horizontales}

En primer lugar, como estudio preliminar se realizó un análisis de contenido para examinar la visibilidad, identidad y accesibilidad de los departamentos de comunicación universitarios en las sedes webs institucionales. Con este estudio se pretendía obtener información sobre las funciones, la estructura, la ubicación en el organigrama de la universidad y los medios de contacto puestos a disposición de los internautas para contactar con estos departamentos.

Los resultados mostraron una visibilidad y accesibilidad limitada, así como carencias en la presentación del servicio que prestan. A pesar de ello permitieron un primer acercamiento al objeto de estudio y fueron fundamentales para comprender su complejidad, así como para diseñar la entrevista. 
Tabla 1. Estructura de la entrevista

\begin{tabular}{|l|l|l|}
\hline \multicolumn{1}{|c|}{ Planificación de la comunicación } & \multicolumn{1}{|c|}{ Departamento de comunicación } & Públicos, acciones y herramientas de comunicación \\
\hline & - Posición en el organigrama universitario & \\
- Objetivos de comunicación & - Funciones & - Públicos \\
- Plan estratégico de comunicación & - Funciones del dircom & - Principales campañas de comunicación \\
- Evaluación de resultados & - Presupuesto & - Canales y herramientas de comunicación \\
& - Contratación externa & \\
\hline
\end{tabular}

Esta fue la siguiente técnica de investigación empleada al considerar que lo más apropiado para conocer a fondo la gestión de la comunicación universitaria era la interlocución directa con los máximos responsables de este cometido.

Frente a un cuestionario cerrado que podría condicionar las respuestas y reducir su riqueza, se empleó la entrevista semiestructurada pues, de acuerdo con Gaitán-Moya y Piñuel-Raigada (1998), plantea preguntas abiertas al entrevistado pero es muy directiva en su desarrollo, además de permitir la selección muestral de los entrevistados y la cuantificación de las respuestas, que pueden ser codificadas a posteriori.

De las 47 universidades objeto de estudio, se seleccionaron aleatoriamente 33 para entrevistar a sus responsables de comunicación. Fijándose una confianza del 10\% (alpha=0,1), el tamaño muestral obtenido es 33, lo cual garantiza un error " $d$ " igual a 0,08 . Esto presupone un margen de error de las estimaciones por debajo del $8 \%$.

Autores como Almansa-Martínez (2011) o Túñez-López (2012) sirvieron de apoyo en el diseño de la entrevista. Las preguntas planteadas a los directores de comunicación ${ }^{3}$ giraban en torno a 3 bloques (tabla 1):

- planificación y estrategia de la comunicación;

- departamento de comunicación;

- públicos, acciones y herramientas de comunicación.

\section{Resultados}

\subsection{Planificación de la comu- nicación}

\subsubsection{Objetivos de comunicación de las universidades públicas}

El $70 \%$ de los directores de comunicación confirma que la gestión de la marca universitaria es clave para transmitir la identidad y los valores de la universidad, alcanzar una mayor visibilidad y notoriedad en su entorno y posicionar competitivamente a su universidad en el mercado universitario. El 24\% enumera además entre los objetivos comunicacionales la proyección internacional de la universidad.

Se sitúa en segundo lugar la reputación, entendida más allá de la imagen, principalmente en términos de liderazgo, pero también de impacto social. El 39,3\% de los entrevistados afirma que la comunicación universitaria busca el reconocimiento y la valoración social para convertirse, o seguir siendo, una universidad de referencia.

La difusión de la actividad institucional, académica y científica (esta última específicamente subrayada por algunos dircoms) de la universidad es objetivo de comunicación para el $33 \%$ de los centros.

\section{De las 47 universidades públicas espa- ñolas sólo 3 consideran la transparencia como un objetivo en la gestión de su co- municación}

La rendición de cuentas es un objetivo comunicacional para el $24 \%$ de las universidades, incluyéndose dentro de este ítem la transparencia (6\%), que no se ha tenido en cuenta por separado debido a su escasa representatividad.

En un $18 \%$, los objetivos comunicacionales se repliegan a los objetivos estratégicos globales universitarios ("el objetivo es servir a la institución para que alcance las metas de su plan estratégico"). Para el mismo porcentaje (18\%), también son objetivos la captación de alumnos y la mejora de la comunicación interna, principalmente para aumentar el sentimiento de pertenencia y lograr una estructura más cohesionada y efectiva, como base fundamental para transmitir una imagen positiva.

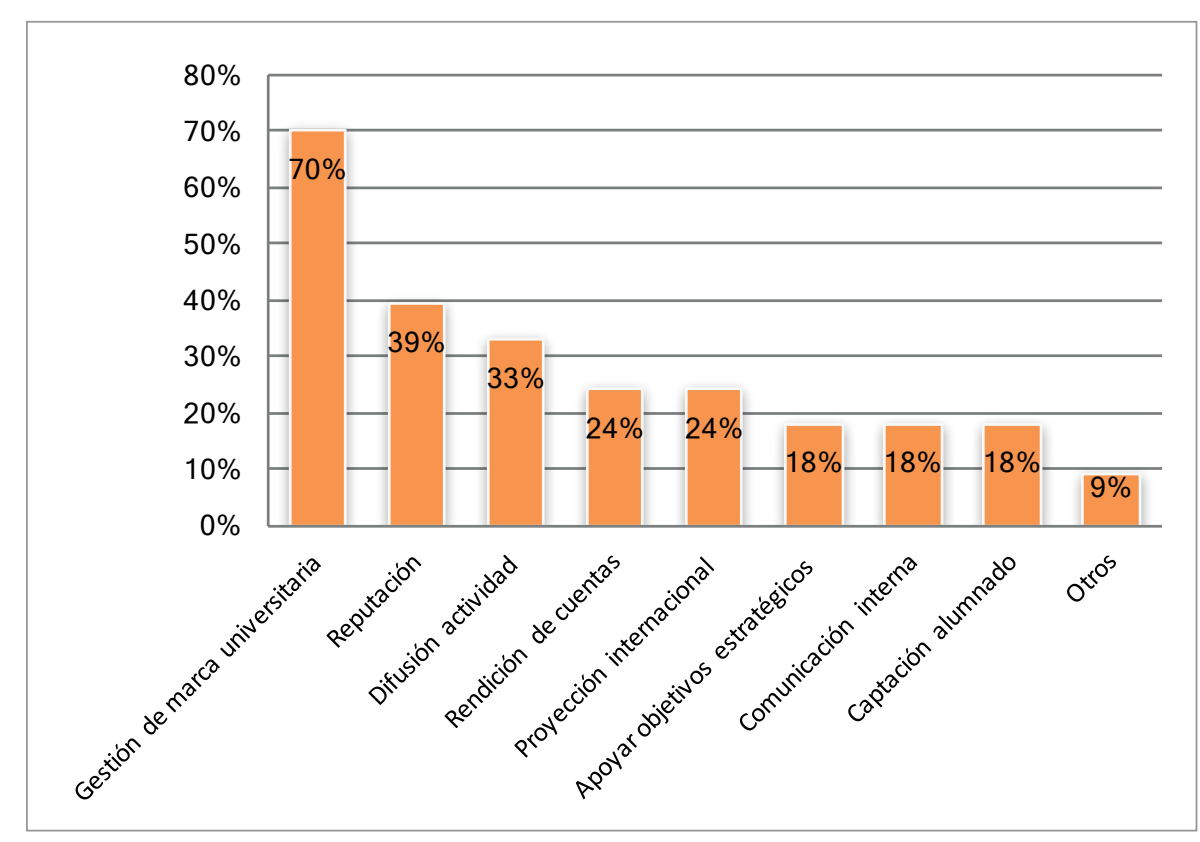

Figura 1. Objetivos de la comunicación universitaria 


\subsubsection{Plan estratégico de comunicación}

El $45 \%$ de los responsables de comunicación declara que su universidad cuenta con un plan estratégico de comunicación y el $55 \%$ declara que no lo tiene. 5 de éstos no explican por qué no tienen; el resto ofrece diferentes respuestas, entre las más representativas estarían:

- se está diseñando uno en el momento de la entrevista (4), bien porque el que existe está obsoleto (3), o porque anteriormente no tenían (1);

- se trabaja directamente partiendo de las directrices comunicacionales recogidas en el plan estratégico universitario (4).

\section{Investigación previa}

El 36\% de las universidades ha implementado una investigación previa para elaborar sus planes de comunicación (pasados, actuales o aún en proceso de elaboración), optándose en el $60 \%$ de los casos por la auditoría de imagen o similares.

\section{Elaboración del plan de comunicación}

Teniendo en cuenta las universidades que tienen un plan de comunicación (14) y las que lo están elaborando (4): la elaboración del plan de comunicación recae en el $61 \%$ de los casos en el responsable de comunicación, o en el equipo de comunicación, que trabaja con o bajo la supervisión del rector o del equipo de gobierno.

\section{Más de la mitad de las universidades pú- blicas no tiene un plan de comunicación}

\subsubsection{Evaluación de los resultados de comunicación}

Para medir los resultados de la actividad comunicativa se usan indicadores cuantitativos (press clippings, visitas a la web y número de seguidores en redes sociales) en un $39,4 \%$ e indicadores de calidad y rendimiento del departamento de comunicación en un $24,2 \%$.

Tres directores admitieron que no se llevaba a cabo ningún

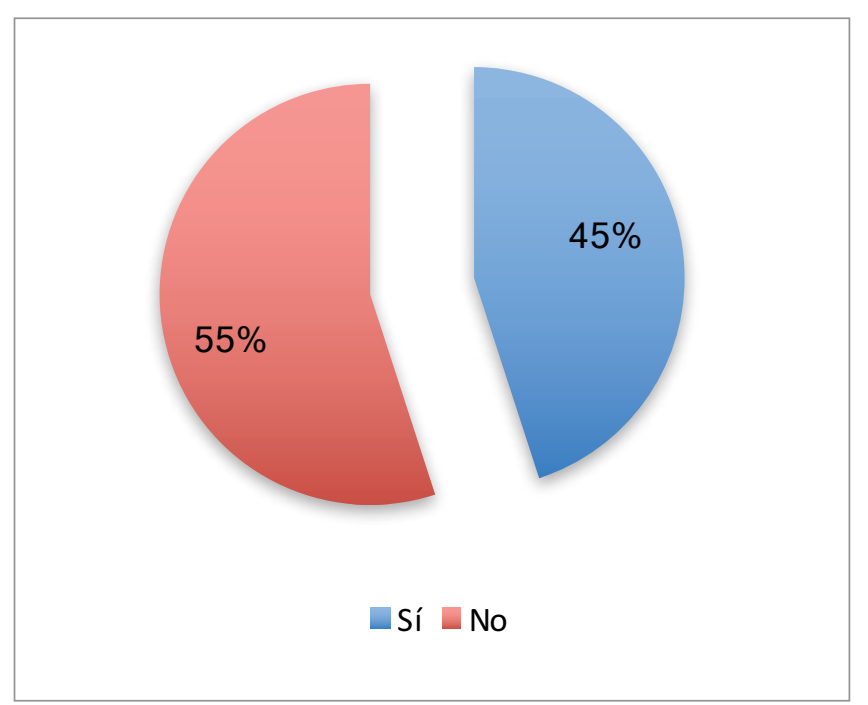

Figura 2. Plan estratégico de comunicación en las universidades públicas tipo de evaluación y, como hecho a destacar, en dos universidades confirmaron que el único modo que tienen de medir los resultados de comunicación es el aumento de matrículas; en otra universidad lo que se hace es preguntar a los estudiantes en las prematrículas.

\subsection{El departamento de comunicación}

\subsubsection{Posición en el organigrama universitario}

El 63,6\% de los departamentos de comunicación depende directamente del rector y el $36,3 \%$ de un vicerrector. En dos casos los directores reseñan que aunque el gabinete dependa de un vicerrector, su figura está vinculada al rector.

\subsubsection{Funciones}

La función más mencionada son las relaciones con los medios (94\%), incluyendo el envío de notas de prensa, atención a los periodistas y otras actividades como "el archivo, la documentación informativa y el monitoreo".

La gestión de medios propios y/o creación y difusión de contenidos y actividades de la universidad a través de medios propios la realiza el $88 \%$ de los departamentos de comunicación. El medio propio que más se ha nombrado y que se considera más importante es la web institucional.

Entre las funciones del departamento de comunicación, el $58 \%$ de los directores nombra la gestión de los perfiles institucionales en las redes sociales, pero hay diferentes grados de profesionalización y aprovechamiento: hay universidades que tienen perfiles institucionales en Facebook y Twitter, pero estos únicamente se usan a modo de tablón de anuncios ("...no interactuamos con la gente porque no tenemos posibilidades de respuesta ni de gestionar las preguntas"), y por el contrario, en una universidad se cuenta incluso con un libro blanco de redes sociales.

Con respecto a la comunicación interna, nombrada como función por dieciséis entrevistados (48\%), citamos una declaración de un director que ejemplifica claramente lo que significa gestión de la comunicación interna dentro de la gestión de la comunicación universitaria:

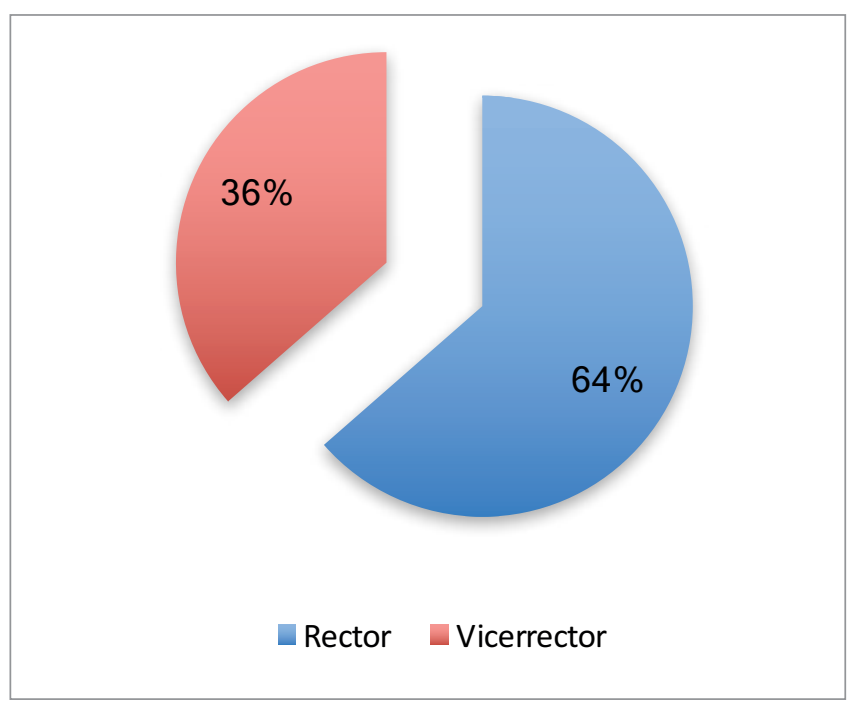

Figura 3. Dependencia del departamento de comunicación en el organigrama universitario 


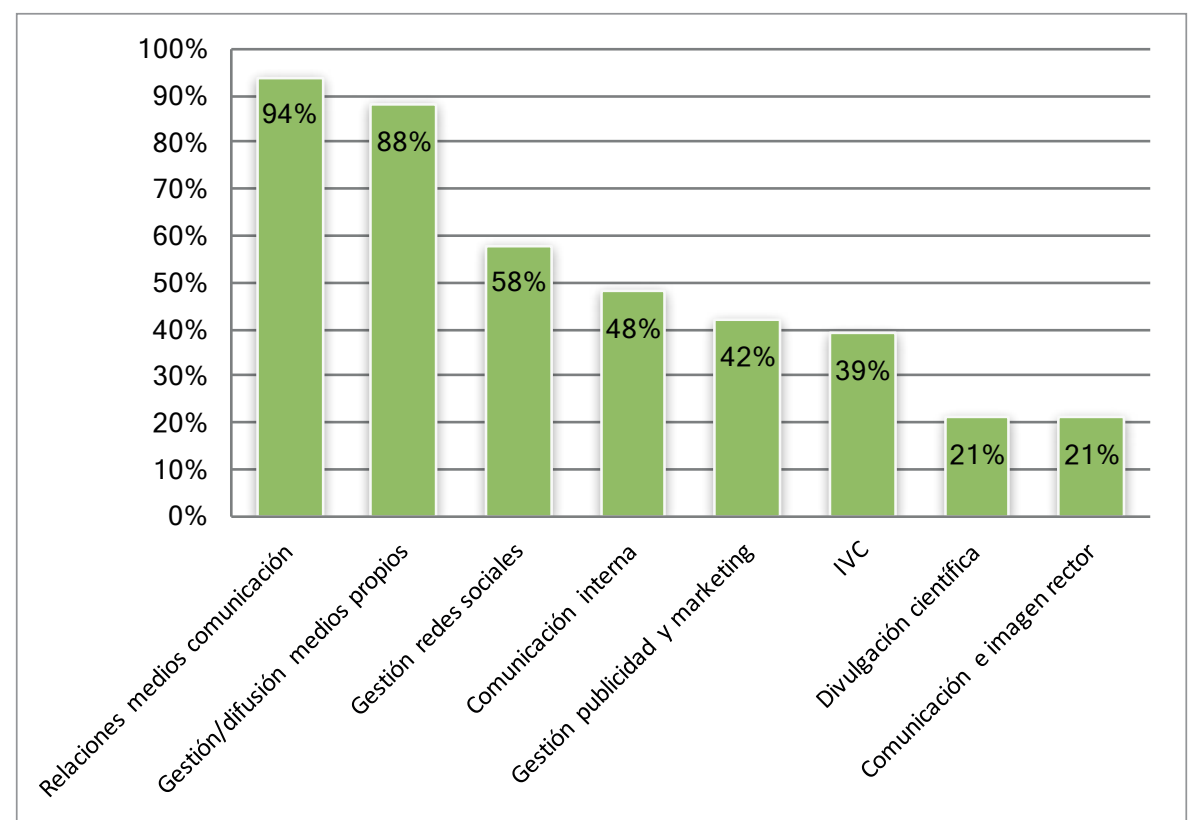

Figura 4. Principales funciones de los departamentos de comunicación universitarios les y de Empleabilidad también gestiona algunos aspectos de comunicación".

"El servicio de información y atención al alumnado se encarga del marketing y las campañas de captación de alumnado (...), la unidad de archivo e imagen está en el departamento de calidad (...)".

Por otra parte, se preguntó a los directores si el departamento de comunicación coordina la comunicación de los diferentes centros universitarios:

En 2 universidades (6\%) existe la figura del delegado de comunicación en los centros que trabaja de forma coordinada con el gabinete.

12 directores $(36,36 \%)$ comentan que desde el gabinete coordinan, o lo intentan, y asesoran en co-

"Cuando hablamos de comunicación interna, hablamos de información o informaciones que afectan a los miembros de la universidad y que nosotros publicamos en medios de comunicación propios de la universidad".

Con un porcentaje menor al $45 \%$, también se realizan funciones como:

- gestión de la publicidad y marketing;

- gestión de la identidad visual corporativa;

- divulgación científica (en algunos casos en coordinación con la unidad de cultura científica);

- asesoramiento en comunicación e imagen al rectorado.

La gestión de la comunicación en las redes sociales evita la interacción con los públicos, usándolas como meros tablones informativos

\subsubsection{Gestión integral y coordinada de la comunicación}

21 directores (el 63,63\%) aseguran que se lleva a cabo una gestión integral de la comunicación, bien sea porque todos los servicios o unidades de comunicación están dentro de un mismo departamento, o porque hay una figura que los coordina.

En 7 universidades (21\%) el área de marketing o el área de imagen son independientes del servicio de comunicación. Aun así, los directores declaran que trabajan de forma coordinada o colaborativa.

En 2 universidades (6\%) explican que se está trabajando para que la comunicación se gestione de forma coordinada y unificada, ya que hasta ahora no se había hecho.

En $3(9 \%)$ sí parece existir una mayor dispersión en la gestión de la comunicación. Por ejemplo:

“(...) además del gabinete, hay un responsable de redes sociales. Y el Vicerrectorado de Relaciones Instituciona- municación a los centros.

En 16 universidades (48,48\%) estos departamentos asesoran a las diferentes instancias universitarias si estas lo requieren.

Otros 2 directores (6\%) dicen que el gabinete es el interlocutor de la universidad en las relaciones con los medios

En un último caso (3\%), un director comenta que:

"Algunas facultades tienen sus propios departamentos de comunicación, pero nosotros ahí no entramos. Nosotros marcamos en qué formato pueden difundir su información".

\subsubsection{El dircom universitario}

En el 51,5\% de las universidades, el dircom juega un papel estratégico, tenga perfil político o técnico, porque diseña y define la estrategia y los objetivos de comunicación con el rector o el

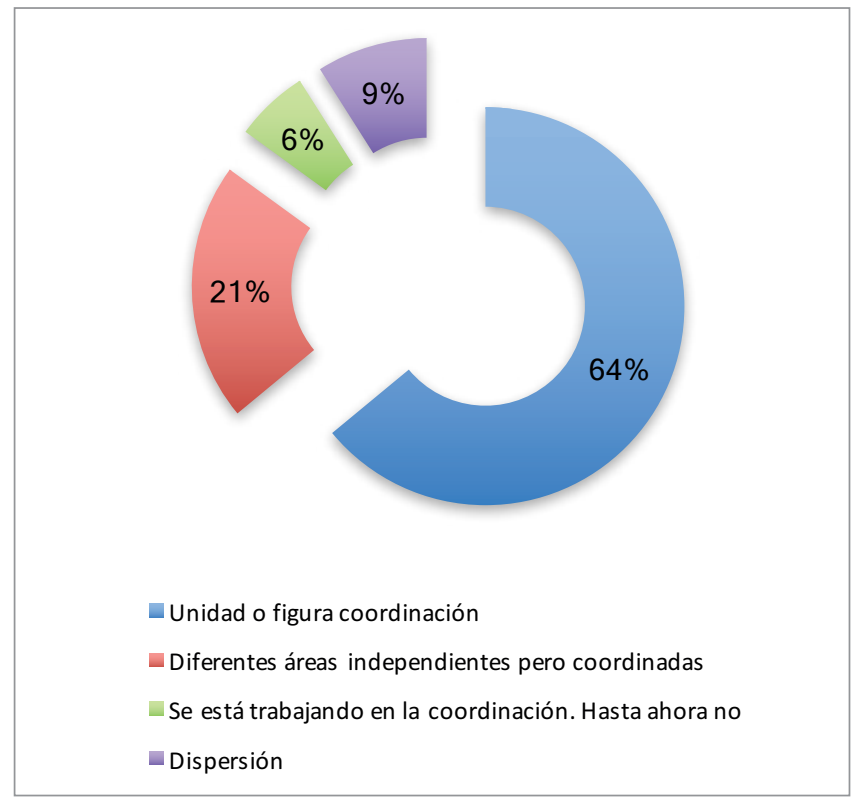

Figura 5. Gestión integral y coordinada de la comunicación 
equipo de gobierno. Otros tres directores no toman decisiones, pero asesoran sobre este tema.

27 directores (81\%) han afirmado que se encargan de gestionar y coordinar al equipo de comunicación, sea este un departamento o sean diferentes áreas. Organizan y supervisan el trabajo.

11 directores (33,33\%) aseguran que también llevan a cabo labores diarias dentro del gabinete, generalmente aquellas de carácter más estratégico, como la atención a los medios, el desarrollo de un briefing o la planificación de una campaña.

Por último, 5 entrevistados (15,15\%) han nombrado entre sus funciones el asesoramiento al rector y otros $3(9 \%)$ aseso-

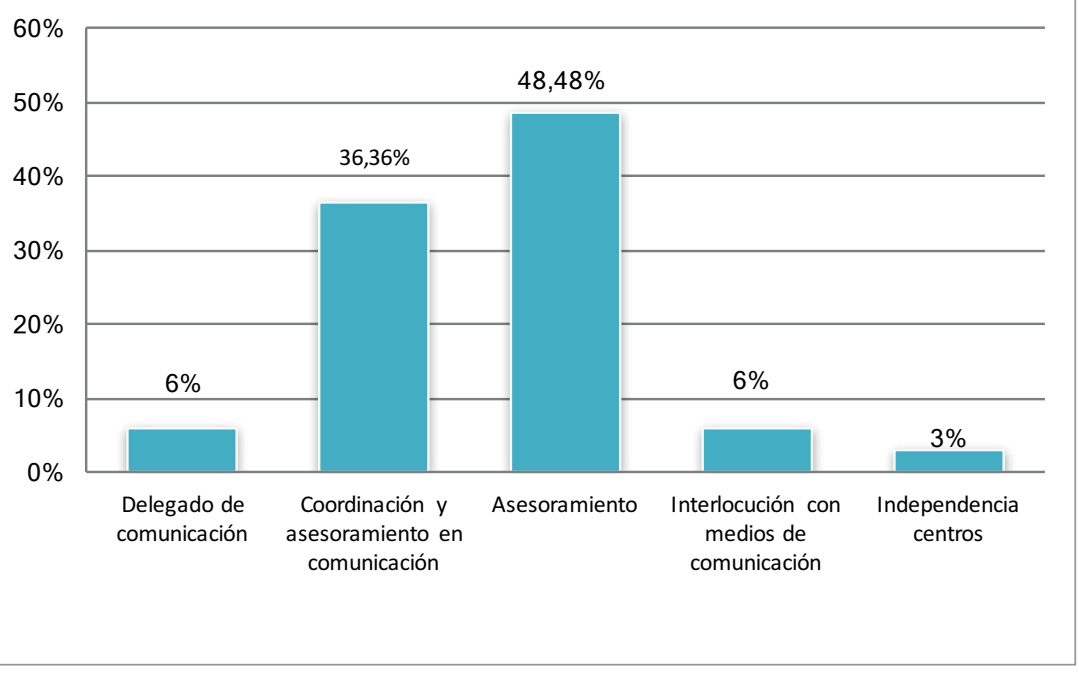

Figura 6. Papel del departamento de comunicación en relación con los centros universitarios ran al equipo de gobierno al completo.

\section{La comunidad universitaria no participa en la toma de decisiones de los planes de comunicación, recayendo esta labor de forma unilateral en el dircom}

\subsubsection{Presupuesto}

Ante las preguntas: “¿el departamento de comunicación tiene un presupuesto determinado?, ¿es posible conocer esa información?", las respuestas fueron esquivas. Sólo 6 directores $(18,18 \%)$ accedieron a decir la partida presupuestaria ${ }^{4}$ con la que cuenta su departamento, mientras que la mayoría $(82 \%)$, no contesta $(21,21 \%)^{5}$ o no concreta $(18,18 \%)$, o remite directamente al portal de transparencia (33,33\%). El $9 \%$ (3) restante afirma que no tienen un presupuesto específico, que éste depende de las necesidades.

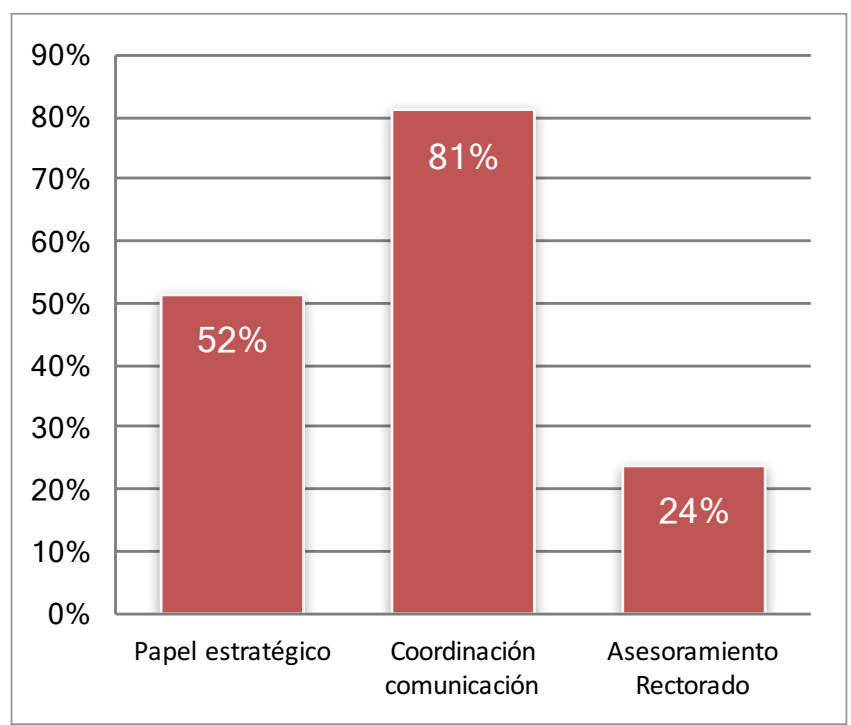

Figura 7. Funciones del dircom universitario
Por otra parte, se han detectado algunos comentarios en las respuestas que hacen referencia a la poca disponibilidad presupuestaria.

\subsubsection{Contratación externa}

En 5 universidades no se subcontrata ningún servicio, porque "lo cubren todo desde el gabinete", aunque también por falta de presupuesto se ajustan a los medios y recursos que tienen. En el resto de los casos se subcontratan servicios de forma puntual.

Los servicios que más se subcontratan son:

- press clipping (39\%);

- publicidad (36\%).

Sea como sea, se insiste en que la creatividad se dirige y supervisa por el dircom o el departamento, o directamente no se contrata.

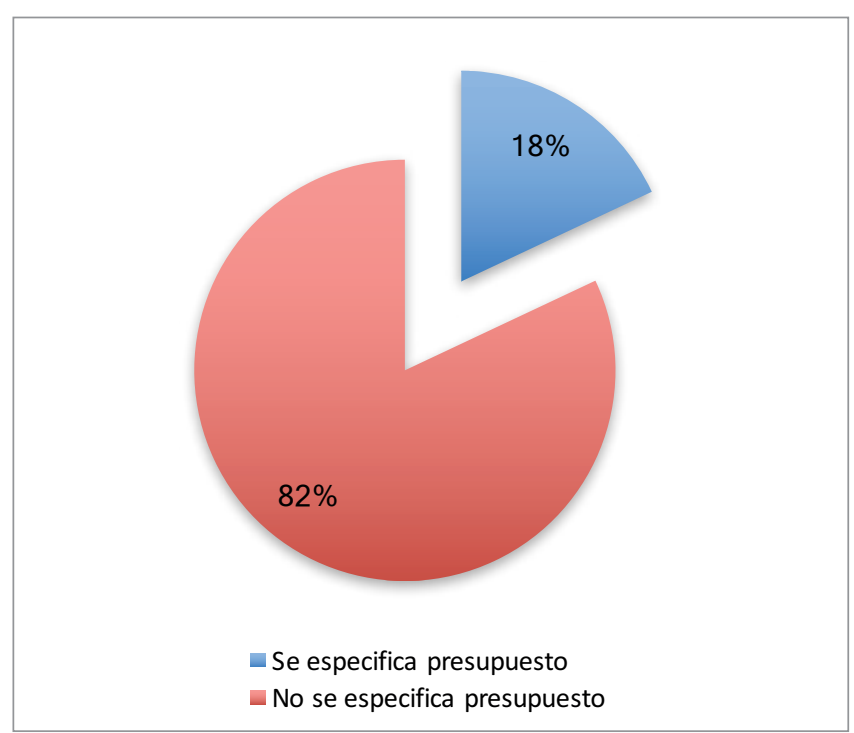

Fgura 8. Directores que especifican el presupuesto del departamento de comunicación 


\subsection{Públicos y canales de la comunicación institucio- nal universitaria}

\subsubsection{Públicos de la comunicación universitaria}

Los públicos con los que se implementan acciones de comunicación desde el departamento de comunicación se muestran en la tabla 2.

Además de citar cada uno de los públicos con los que se trabaja desde estos departamentos, algunos dircoms hicieron comentarios a esta pregunta:

"Con todos los públicos. Lo primero que hicimos al llegar fue detectar los públicos de la organización (133 públicos organizados en 21 grupos principales), para determinar la estrategia para dar a conocer la universidad".

"Con todos los stakeholders: mapa de públicos convencional".

“Hacia quién comunicamos depende más del target de los medios que usamos en cada caso que no de nuestra voluntad".

"Primero nos debemos a nuestros clientes, que son los que pagan las matrículas más altas de España. En segundo lugar, a las familias. En tercer lugar, a la comunidad universitaria; en cuarto a las empresas y en quinto, a la sociedad en general".

"Toda la universidad se mueve y se relaciona con los públicos. Nosotros no monopolizamos ninguno, posiblemente los medios de comunicación. En la relación con los demás públicos, hay participación de más gente".

\subsubsection{Campañas}

Se les preguntó a qué públicos dirigen más campañas y con qué objetivos. De los 20 directores que contestaron ${ }^{6}$, el $85 \%$ mencionó las campañas de captación de alumnado, de grado y posgrado, asegurando que los futuros estudiantes, el alumnado y los egresados son los colectivos a los que se dirigen más campañas, fundamentalmente para lograr un mayor número de matrículas.

Los estudiantes potenciales son el único público al que se dirigen campañas de marketing y publicidad, con el objetivo de aumentar las matrículas

\subsubsection{Canales y medios de comunicación}

El $85 \%$ de los entrevistados ha citado la web institucional como canal de comunicación con sus públicos externos e internos. Según el testimonio de muchos de ellos es el canal más importante.

Los perfiles institucionales en redes sociales, principalmente Facebook y Twitter, son el segundo medio más citado (81\%), también como canal de comunicación interna y externa. En cinco ocasiones han aceptado que deberían mejorar y pro-
Tabla 2. Públicos de la comunicación universitaria

\begin{tabular}{|l|c|}
\hline \multicolumn{1}{|c|}{ Público } & $\mathbf{n}$ \\
\hline Públicos internos (PDI; PAS; alumnado) & 28 \\
\hline Medios de comunicación & 23 \\
\hline Futuros estudiantes & 18 \\
\hline Sociedad o público general & 16 \\
\hline Empresas & 14 \\
\hline Otras instituciones y administraciones (locales, regionales, & 12 \\
\hline nacionales e internacionales) & 7 \\
\hline Egresados o alumni & 4 \\
\hline Padres o familias de futuros alumnos & 3 \\
\hline Públicos externos (no se especifica cuáles) & 3 \\
\hline Otras universidades & 2 \\
\hline Asociaciones & 2 \\
\hline Colegios y colectivos profesionales & 2 \\
\hline Orientadores y prescriptores de secundaria & 2 \\
\hline Patrocinadores que colaboran con la actividad universitaria & 1 \\
\hline Institutos de investigación & 1 \\
\hline Fundaciones en las que participa la universidad & 1 \\
\hline Líderes de opinión & 2 \\
\hline Partidos políticos & 2 \\
\hline & 2 \\
\hline
\end{tabular}

fesionalizar su gestión y un director ha afirmado que tienen perfiles institucionales en Facebook y Twitter "pero que no interactúan".

Los medios de comunicación tradicionales son el tercer canal más nombrado por los entrevistados (75\%), "esenciales para tener repercusión en la sociedad en general". De acuerdo con los directores, las universidades mantienen relaciones asiduas con los medios de comunicación, especialmente con la prensa; se les envía la información que se genera en la universidad, se les convoca a actos institucionales y se pone a los periodistas en contacto con expertos.

Mientras el correo electrónico o las listas de correo son citadas por 8 directores $(24,24 \%)$ como herramienta para informar de las actividades de la universidad, la newsletter o los boletines de noticias van ganando terreno, al ser nombrados en 14 ocasiones $(42,42 \%)$ precisamente para esta tarea, notificándose únicamente de forma diferenciada noticias de primer orden para no saturar al destinatario.

En 7 casos $(21,21 \%)$ se hace alusión a las revistas institucionales universitarias, que tienen "funciones de comunicación interna amplia".

El portal de comunicación web, la sala de prensa 2.0 o el canal de noticias del gabinete en la propia web institucional son para 6 directores $(18,18 \%)$ otro de los canales de comunicación tanto con públicos internos como externos.

Otros canales, nombrados en menor medida, son la televisión universitaria, la radio universitaria o el portal de transparencia. 


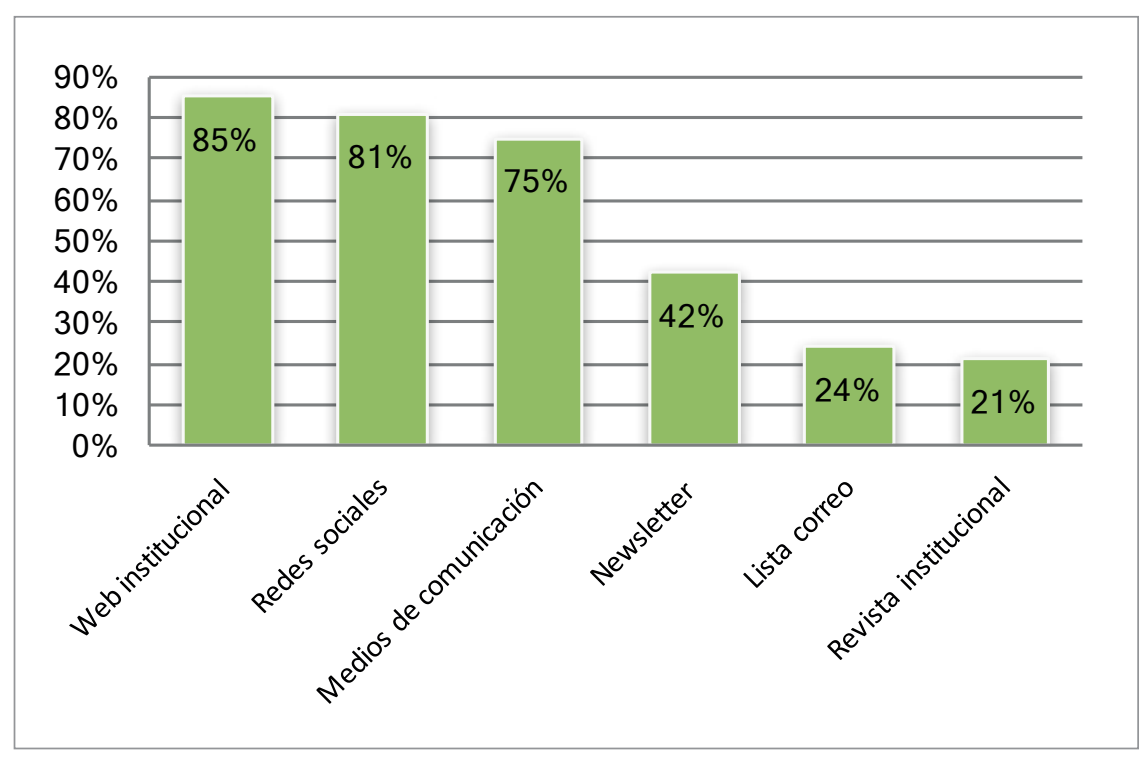

Figura 9. Principales canales y medios de comunicación ha comprobado que la comunidad universitaria no participa en la toma de decisiones de los planes de comunicación, y cuando lo hace es de forma consultiva, recayendo esta labor de forma unilateral en el dircom que trabaja con, o bajo la supervisión del equipo de gobierno.

En relación con los centros universitarios, el papel del departamento de comunicación es ofrecer asesoramiento si se le requiere, y en todo caso, ejercer de interlocutor en las relaciones con los medios.

Entre las funciones principales de los departamentos de comunicación de las universidades públicas prevalecen, casi en igualdad de condiciones, las relaciones con los medios de comunicación y la gestión de in-

\section{Discusión de resultados}

A pesar de la importancia capital de la planificación estratégica para una gestión eficaz de la comunicación, los resultados obtenidos muestran que más de la mitad de las universidades públicas no tiene un plan de comunicación. También se observan graves déficits en cuanto a la evaluación de la actividad comunicativa. Según las explicaciones ofrecidas, el volumen y la urgencia del trabajo diario, el bajo presupuesto destinado a comunicación o la escasa importancia que se le da a esta en la gestión universitaria, podrían ser las causas más determinantes para que esto se produzca.

Los resultados relativos a la dependencia orgánica del departamento de comunicación (el 63,6\% depende del rector y el $36,3 \%$ de un vicerrectorado) no continúan la tendencia detectada por Durán-Mañes (2005), según la cual los departamentos de comunicación tenderían a depender cada vez en mayor medida de vicerrectorados. Se demuestra de este modo que la ubicación de los servicios de comunicación puede cambiar cuando existe una renovación del equipo del gobierno, hecho que no ocurre con otras áreas.

El objetivo prioritario de comunicación de las universidades públicas es impulsar o mejorar su imagen y/o su reputación, para alcanzar una mayor visibilidad y notoriedad y lograr una posición de liderazgo en su entorno y cada vez más a nivel internacional, siendo la proyección internacional otro de los objetivos comunicacionales que va adquiriendo más peso. Este hecho supone la asunción por parte de las universidades públicas del modelo de gestión comunicativa corporativa, cuestión que desde nuestro punto de vista no es ajena al contexto competitivo, las últimas reformas legislativas y la reducción presupuestaria a la que se está viendo sujeta la educación superior (Simancas-González; García-López, 2016).

En comunicación interna se puede afirmar que no se gestiona desde un enfoque global e integral en consonancia con la comunicación externa y que el cometido de los servicios de comunicación es únicamente el de ejercer de transmisores de información institucional y del gobierno universitario. Se formación y contenidos a través de medios propios, aunque consideramos que esta última va a ganar terreno en un breve plazo de tiempo. Sin embargo, es necesario destacar que al menos hasta ahora esta apuesta no ha significado más que un trasvase al entorno digital, no habiéndose modificado ni el tipo de información ofrecido por la institución ni los procesos comunicativos entre ésta y sus diferentes públicos.

Los gabinetes de comunicación asumen otras funciones, como la gestión de la publicidad y el marketing o la gestión de los perfiles en redes sociales. Asimismo adquiere importancia la divulgación científica, y se prevé que pueda convertirse en una de las prioridades de la comunicación universitaria.

Las tareas relacionadas con la comunicación no siempre las llevan a cabo instancias aglutinadas en una sola área, incluso en algunas universidades los órganos que implementan funciones de comunicación pueden depender de diferentes vicerrectorados. A pesar de ello, más del $90 \%$ de los directores recalca que se trabaja de forma colaborativa o coordinada, siendo ellos mismos los encargados de esta tarea. La tendencia parece ser la creación de una única área de comunicación que englobe todas las funciones relacionadas con la comunicación.

El portal web institucional se consolida como el canal principal para difundir información sobre la institución y para la transmisión de los valores de la marca universitaria, concordando esto último con las conclusiones de Blanco-Sánchez (2014).

Podríamos decir que la comunicación universitaria pivota entre dos públicos:

- los futuros estudiantes, en sentido amplio y no sólo como
potenciales estudiantes de grado;
- los medios de comunicación.

Tienen cada vez más importancia los primeros, siendo el único público de la comunicación universitaria al que se dirigen campañas de marketing y publicidad con el objetivo de aumentar las matrículas, pues de ello depende que mejore en 
parte la situación de déficit de las universidades españolas. Esto es sintomático del giro que se está produciendo en estos departamentos: de las relaciones con los medios a la comunicación corporativa y la creación de marcas universitarias.

\section{Conclusiones}

Además de los grandes déficits detectados en la planificación estratégica de la comunicación y la escasa estabilidad estratégica y de continuidad en la gestión de la comunicación universitaria, el hecho de que las estrategias se centren en la proyección de imagen y marca universitaria (que se advierte en la tendencia de un mayor enfoque hacia la comunicación corporativa) revela que prima un modelo de comunicación instrumental y finalista, guiado por la competitividad y la racionalidad empresarial (Quirós-Fernández, 2016), primando los criterios cuantitativos. Todo esto cobra pleno sentido en el proceso de transformación estructural en el que está inmersa la universidad pública española (Díez-Gutiérrez et al., 2010).

Se detecta una tendencia hacia la gestión de los medios propios, usados hasta ahora como herramientas y canales de comunicación unidireccionales y verticales, a nivel interno y externo, lo que parece evidenciar un modelo difusionista, refractario a la bidireccionalidad y a claves diálogicas ${ }^{7}$. Esto se refuerza con el hecho de que los agentes universitarios y los grupos de interés se entienden exclusivamente como receptores pasivos, como simples destinatarios de mensajes.

Atendiendo al carácter público y la función social de la institución, consideramos que sería necesaria, y seguramente más eficaz en términos de imagen, la búsqueda de soluciones comunicativas que promuevan el diálogo con los agentes externos y fomenten la participación activa de la comunidad universitaria.

\section{Notas}

1. Según el Registro de Universidades, Centros y Títulos del Ministerio de Educación hay un total de 85 universidades en el estado español: 35 de titularidad privada y 50 públicas (última consulta: 15/02/2017).

2. Universidad Internacional de Andalucía, Universidad Internacional Menéndez Pelayo y Universidad Nacional de Educación a Distancia.

3. Las entrevistas fueron telefónicas, de una duración aproximada de 1 hora. Se llevaron a cabo durante los meses de mayo, junio y julio de 2015.

4. Los presupuestos manejados van desde los 35.000 a los 310.000 euros. No hemos comprobado las funciones que tiene asignadas cada uno de estos departamentos, pues al ser tan pocos los que han accedido a darnos la cifra la comparación carece de interés.

\section{4 admiten que no quieren o no pueden dar la cifra.}

6. 13 directores no respondieron a la pregunta porque explicaron directamente las acciones de comunicación que llevaban a cabo con los públicos (siguiente cuestión).

7. De hecho, ningún entrevistado hizo mención alguna a estas claves.

\section{Bibliografía}

Almansa-Martínez, Ana (2011). Del gabinete de prensa al gabinete de comunicación. La dirección de comunicación en la actualidad. Zamora: Comunicación Social. ISBN: 97884 92860913

Blanco-Sánchez, Tania (2014). La comunicación online de las marcas universitarias españolas en sus sedes webs. Tesis doctoral. Universidad de Extremadura.

http://dehesa.unex.es/handle/10662/1554

Castillo-Díaz, Ana (2007). La comunicación de las marcas universitarias españolas a través de sus sedes webs institucionales. Tesis doctoral. Universidad de Extremadura.

Chomsky, Noam (2013). "Sobre el trabajo académico, el asalto neoliberal a las universidades y cómo debería ser la educación superior". Bajo el volcán, v. 13, n. 21, pp. 121134.

http://www.redalyc.org/articulo.oa?id=28640302006

De-Aguilera-Moyano, Miguel; Farias-Batlle, Pedro; Baraybar-Fernández, Antonio (2010). "La comunicación universitaria: modelos, tendencias y herramientas para una nueva relación con sus públicos". Icono 14: Revista de comunicación y nuevas tecnologías, v. 2, n. 3, pp. 90-124. https://doi.org/10.7195/ri14.v8i2.248

Díez-Gutiérrez, Enrique J.; Guamán, Adoración; Jorge-Alonso, Ana; Ferrer-Llop, Josep (2014). Qué hacemos con la universidad. Madrid: Akal. ISBN: 9788446039082

Durán-Mañes, Ángeles (2005). Nuevas tendencias en la comunicación corporativa: Aplicación de un modelo de corporate universitario. Tesis doctoral. Universitat Jaume I. ISBN: 9788469318706

http://www.tdx.cat/handle/10803/10472

Fernández-Beltrán, Francisco (2007). La gestión de la nueva comunicación interna. Análisis de la aplicación de las tecnologías de la información en los procesos de comunicación interna de las universidades de la Comunidad Valenciana. Tesis doctoral. Universitat Jaume I. ISBN: 9788469075651 http://www.tesisenred.net/handle/10803/10464

Gaitán-Moya, Juan-Antonio; Piñuel-Raigada, José-Luis (1998). Técnicas de investigación en comunicación social. Madrid: Editorial Síntesis. ISBN: 8477386048

Gómez-Calderón, Bernardo-José; Paniagua-Rojano, Francisco-Javier (2014). "Las universidades españolas en Twitter: mensajes, contenidos y públicos". Historia y comunicación social, v. 19, pp. 681-694.

https://doi.org/10.5209/rev_HICS.2014.v19.44994

González-Díaz, Cristina; Iglesias-García, Mar; Codina, Lluís (2015). "Presencia de las universidades españolas en las redes sociales digitales científicas: caso de los estudios de comunicación". El profesional de la información, v. 24, n. 5, pp. 640-647.

https://doi.org/10.3145/epi.2015.sep.12

Herranz-de-la-Casa, José-María; Tapia-Frade, Alejandro; Vicente-Lázaro, Arturo (2009). "La comunicación interna en la universidad. Investigar para conocer a nuestros públicos". Revista latina de comunicación social, n. 64, pp. 262-274. 
https://doi.org/10.4185/RLCS-64-2009-822-262-274

López-Pérez, Lourdes; Olvera-Lobo, María-Dolores (2016). "Comunicación pública de la ciencia a través de la web 2.0. El caso de los centros de investigación y universidades públicas de España". El profesional de la información, v. 25, n. 3, pp. 441-448.

https://doi.org/10.3145/epi.2016.may.14

Losada-Díaz, José-Carlos (2002). Prensa e imagen corporativa en la Universidad. Murcia: Ucam-Aedos. ISBN: 97884 95383297

Losada-Díaz, José-Carlos (2004). “La comunicación en la construcción de marcas universitarias". En: Losada-Díaz, Juan-Carlos (coord.). Gestión de la comunicación en las organizaciones: comunicación interna, corporativa y de marketing. Barcelona: Arial Comunicación, pp. 475-490. ISBN: 8434413035

Losada-Vázquez, Ángel (1998). La comunicación institucional en la gestión del cambio: el modelo universitario. Salamanca: Publicaciones Universidad Pontificia. ISBN: 97884 72994133

Palencia-Lefler-Ors, Manuel (2008). "La incomunicación interna en la Universidad española". Revista latina de comunicación social, n. 63, pp. 277-286.

https://doi.org/10.4185/RLCS-63-2008-766-277-286

Paniagua-Rojano, Francisco-Javier; Gómez-Calderón, Bernardo-José (2012). "Hacia la comunicación 2.0. El uso de las redes sociales por parte de las universidades españolas". Icono 14: revista de comunicación y tecnologías emergentes, v. 10, n. 3, pp. 346-364.

https://doi.org/10.7195/ri14.v10i3.473
Paniagua-Rojano, Francisco-Javier; Gómez-Calderón, Bernardo-José; Fernández-Sande, Manuel (2012). "La incorporación de los departamentos de comunicación de las universidades españolas al entorno digital. Un análisis cuantitativo". Estudios sobre el mensaje periodístico, v. 18, pp. 691-701. http://dx.doi.org/10.5209/rev_ESMP.2012.v18.40948

Quirós-Fernández, Fernando-José (2016). "La universidad gerencial en Europa y los procedimientos de evaluación de la 'calidad' de la docencia y la investigación en España". Chasqui. Revista latinoamericana de comunicación, n. 133, pp. 191-208. https://doi.org/10.16921/chasqui.v0i133.2922

Simancas-González, Esther (2016). La comunicación en la universidad pública española. De la comunicación instrumental a la comunicación para la democracia y la participación. Tesis doctoral. Universidad de Málaga.

http://riuma.uma.es/xmlui/handle/10630/12246

Simancas-González, Esther; García-López, Marcial (2016). "Historia de un secuestro: de la Iglesia a la marca. Evolución histórica de la universidad en España". Chasqui. Revista latinoamericana de comunicación, n. 133, pp. 173-190.

http://dx.doi.org/10.16921/chasqui.v0i133.2953

Túñez-López, Miguel (2012). La gestión de la comunicación en las organizaciones. Zamora: Comunicación Social. ISBN: 9788492860937

Zamora-Medina, Rocío; Hernández-Gómez, Francisco (2014). "La triangulación interdisciplinar como propuesta metodológica para el estudio de la imagen pública de las universidades". Sphera publica, v. 1, n. 14, pp. 39-69. http://sphera.ucam.edu/index.php/sphera-01/article/ download/168/200

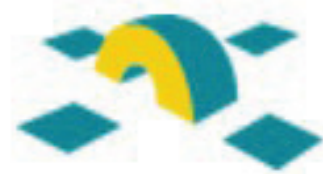
चा RIS

\section{COMUNICACIÓN}

\section{https://www.rediris.es/list/info/comunicacion.htm/}

Comunicación es una lista de distribución en castellano para debatir y estar al día sobre teoría de la comunicación, comunicación política, comunicación industrial, relaciones públicas, comunicación audiovisual y multimedia, radio y televisión, cinematografía, periodismo, periodismo de datos, divulgación de la ciencia, medios y cibermedios, redes sociales... y todos los aspectos relacionados con la COMUNICACIÓN.

Empezó a funcionar en enero de 2017 y está alojada en el servicio de listas de RedIRIS, desde donde es posible consultar sus archivos:

https://listserv.rediris.es/cgi-bin/wa?AO=COMUNICACION

La lista cuenta con 2 moderadores que permanentemente filtran los mensajes para evitar spam, mensajes inapropiados, anuncios, mensajes repetidos, etc.:

Isabel Olea (EPI, León)

Tomàs Baiget (EPI, Barcelona)

Puedes suscribirte a Comunicación en:

https://listserv.rediris.es/cgi-bin/wa?SUBED1=COMUNICACION\&A=1 